\title{
Three-Dimensional Comparison of Ultrastructural Characteristics at Depressing and Facilitating Synapses onto Cerebellar Purkinje Cells
}

\author{
Matthew A. Xu-Friedman, ${ }^{1}$ Kristen M. Harris, ${ }^{2}$ and Wade G. Regehr ${ }^{1}$ \\ ${ }^{1}$ Department of Neurobiology, Harvard Medical School, Boston, Massachusetts 02115, and 2Department of Biology, \\ Boston University, Boston, Massachusetts 02215
}

Cerebellar Purkinje cells receive two distinctive types of excitatory inputs. Climbing fiber (CF) synapses have a high probability of release and show paired-pulse depression (PPD), whereas parallel fiber (PF) synapses facilitate and have a low probability of release. We examined both types of synapses using serial electron microscopic reconstructions in 15-d-old rats to look for anatomical correlates of these differences. PF and CF synapses were distinguishable by their overall ultrastructural organization. There were differences between PF and CF synapses in how many release sites were within $1 \mu \mathrm{m}$ of a mitochondrion (67 vs $84 \%$ ) and in the degree of astrocytic ensheathment (67 vs $94 \%$ ). However, the postsynaptic density sizes for both types of synapses were similar $\left(0.13-0.14 \mu \mathrm{m}^{2}\right)$. For both types of synapses, we counted the number of docked vesicles per release site to test whether this number determines the probability of release and synaptic plasticity. PF and CF synapses had the same number of anatomically docked vesicles (7-8). The number of docked vesicles at the CF does not support a simple model of PPD in which release of a single vesicle during the first pulse depletes the anatomically docked vesicle pool at a synapse. Alternatively, only a fraction of anatomically docked vesicles may be release ready, or PPD could result from multivesicular release at each site. Similarities in the number of docked vesicles for PF and CF synapses indicate that differences in probability of release are unrelated to the number of anatomically docked vesicles at these synapses.

Key words: climbing fiber; parallel fiber; probability of release; docked vesicles; electron microscope; serial reconstruction
Purkinje cells receive two kinds of excitatory synaptic inputs (Palay and Chan-Palay, 1974), each of which exhibits different short-term plasticity and initial probability of release. The first originates in the inferior olive and is known as the climbing fiber (CF) (Ramón y Cajal, 1995). The CF shows prominent pairedpulse depression (PPD) (Eccles et al., 1966; Hashimoto and Kano, 1998) and has a high probability of release, which has been estimated at $>40 \%$ (Dittman and Regehr, 1998; Silver et al., 1998). The second input is from cerebellar granule cells, which send their axons into the cerebellar molecular layer as parallel fibers (PFs). PFs show prominent paired-pulse facilitation (Konnerth et al., 1990; Perkel et al., 1990) and have a low probability of release, estimated to be $<5 \%$ (Dittman et al., 2000).

The differences in short-term plasticity and initial probability of release between PFs and CFs are important because they are the only excitatory inputs to Purkinje cells, which are themselves the only outputs from the cerebellar cortex. However, the mechanisms underlying short-term plasticity and the probability of release are not well understood. CF and PF synapses present a useful system with which to examine these issues because they

Received May 7, 2001; revised June 15, 2001; accepted June 19, 2001.

This work was supported by National Institutes of Health Grants NS07112 (W.G.R.) and NS21184 and MH57351 (K.M.H.). We thank M. Feinberg, M. Ericsson, and E. Benecchi for invaluable help with the electron microscopy and J. Fiala for help using the SEM Align and IGL Trace programs. K. Foster and A. Kreitzer provided help with the fluorescent labeling. We thank D. Blitz, A. Carter, K. Foster, A. Kreitzer, and K. Vogt for comments on this manuscript.

Correspondence should be addressed to Wade G. Regehr, Department of Neurobiology, Harvard Medical School, 220 Longwood Avenue, Boston, MA 02115. E-mail: wade_regehr@hms.harvard.edu.

Copyright (ㄷ) 2001 Society for Neuroscience $0270-6474 / 01 / 216666-07 \$ 15.00 / 0$ have different properties while synapsing onto the same target neuron.

One approach to understanding physiological differences between synapses has been to compare their anatomical structures (Herrera et al., 1985; Walrond et al., 1993; Msghina et al., 1998; Schikorski and Stevens, 1999). One hypothesis for the basis of the probability of release is that it is proportional to the number of docked vesicles. This hypothesis predicts that the number of docked vesicles at $\mathrm{CF}$ release sites would be markedly greater than at PF release sites, because of the extreme differences between them in probability of release. Studies at other synapses have found a correlation between the probability of release and the size of the readily releasable pool (RRP) of vesicles, as quantified using physiological stimulation (Stevens and Tsujimoto, 1995; Dobrunz and Stevens, 1997), FM1-43 staining (Murthy et al., 1997), and by counting docked vesicles (Harris and Sultan, 1995; Schikorski and Stevens, 1997, 1999, 2001).

Another aspect of short-term plasticity to be explained is the mechanism underlying PPD. PPD has been proposed to result from a reduction in the number of release-ready synapses through depletion of docked vesicles (Takeuchi, 1958; Elmqvist and Quastel, 1965; Betz, 1970; Dobrunz and Stevens, 1997). CFs show strong PPD, so measurements of the number of docked vesicles will provide useful information for depletion models.

We used serial electron microscopy (EM) to make detailed reconstructions of both $\mathrm{CF}$ and PF synapses onto Purkinje cells. Our primary focus was on features that may affect short-term plasticity, but we have also documented characteristics relevant to other aspects of synaptic physiology. Although we found some differences, PFs and CFs had similar numbers of docked vesicles, sizes of postsynaptic density (PSD), and postsynaptic spine vol- 
umes. Both fibers ended exclusively on spines, and the release sites were predominantly ensheathed by astrocytes. These data constrain models concerning the mechanisms underlying PPD. In addition, they suggest that the difference in the probability of release between PFs and CFs cannot be accounted for by the number of docked vesicles.

\section{MATERIALS AND METHODS}

Two 15-d-old Long-Evans hooded rats (weights of 28 and $31 \mathrm{gm}$ ) were used for the reconstructions. Each animal was heavily anesthetized using Nembutal $(0.8 \mathrm{mg} / 10 \mathrm{gm})$ and was perfused transcardially for $30 \mathrm{~min}$ with a $2 \%$ paraformaldehyde $-2.5 \%$ glutaraldehyde mix in $0.1 \mathrm{M}$ cacodylate buffer (CB), $\mathrm{pH} 7.3$, warmed to $34-37^{\circ} \mathrm{C}$. After $1 \mathrm{hr}$, the brain was removed and stored in the same fixative at room temperature overnight. Parasagittal thick sections $(400 \mu \mathrm{m})$ were prepared on a tissue chopper, fixed with $1 \% \mathrm{OsO}_{4}-1.5 \% \mathrm{KFeCN}$ in $\mathrm{CB}$, subsequently fixed with $1 \%$ $\mathrm{OsO}_{4}$ in $\mathrm{CB}$, and stained with $1 \%$ uranyl acetate before dehydrating through acetone and embedding in 1:1 Epon/Spurr's resin. Serial thin sections were cut on a Reichert Ultracut S (Leica, Nussloch, Germany), collected on Formvar-coated slot grids, dipped in saturated uranyl acetate in acetone, rinsed in water, dipped in $0.2 \%$ lead citrate, rinsed, and dried.

The effects of tissue preparation on synaptic ultrastructure are not completely known. Studies comparing aldehyde fixation and rapid freezing do report differences in vesicle and mitochondrion shape (Nakajima and Reese, 1983; Brewer and Lynch, 1986), but importantly, differences were not reported for the synaptic characteristics we measured here. There are conflicting reports of the effects of aldehyde fixation on synaptic release (Smith and Reese, 1980; Rosenmund and Stevens, 1997), but its easier applicability in vivo made it the preferable method. We subsequently did not observe evidence of significant vesicular release, such as large numbers of $\Omega$ profiles at the synapses of interest.

Three series of sections were prepared and photographed on a Jeol (Peabody, MA) 1200EX electron microscope with the grids mounted in a rotating holder (SRH $10 \mathrm{mod}$ ). The accelerating voltage was $80-120 \mathrm{kV}$ at a magnification of 5000-10,000 $\times$. Magnification was calibrated using diffraction grating replica number 60021 (Ernest F. Fullam Inc., Latham, NY). Section thickness was calibrated using cylindrical objects such as dendrites and mitochondria (Fiala and Harris, 2001b). Photographic negatives were scanned at 1000 dots per inch on an AGFA (Ridgefield Park, NJ) Duoscan 2500, and the resulting images were aligned using SEM Align (available from the "Tools" section of website www.synapses. bu.edu), by identifying several pairs of matching points in adjacent sections and using a least-squares linear fit (Fiala and Harris, 2001a).

Synapses were selected for reconstruction on the basis of their completeness and optimal angle of sectioning (i.e., perpendicular to the synaptic cleft). Relevant structures were traced and measured with IGL Trace (www.synapses.bu.edu). When identifying docked vesicles, image contrast and brightness were optimized to detect cytoplasmic space between the vesicle and presynaptic membranes. For display purposes, object surfaces were exported from IGL Trace to trueSpace 4.3 (Caligari Corp., Mountain View, CA) for three-dimensional (3-D) rendering. To examine an extensive segment of a $\mathrm{CF}$, up to four photographs per section were arranged into a composite using Adobe Photoshop 5.5 (Adobe Systems, San Jose, CA) before aligning. Figures 2 and 5 were colorized using Igor 4 (WaveMetrics Inc., Lake Oswego, OR).

We also labeled PFs and CFs with fluorescent dyes. To label PFs, we prepared transverse $300 \mu \mathrm{m}$ slices from rat cerebellar vermis and directed a jet of dye $(0.06 \%$ Fast Green, $0.06 \%$ Triton X-100, and $0.09 \%$ 10,000 molecular weight Texas Red dextran in $\mathrm{H}_{2} \mathrm{O}$ ) at the molecular layer, with a large suction pipette placed nearby to restrict the area labeled. We waited at least $1 \mathrm{hr}$ for the dye to diffuse before imaging fibers $>100 \mu \mathrm{m}$ from the fill site. To label CFs, we injected $0.5 \mu \mathrm{l}$ of dye (saturated DiI in dimethylformamide-DMSO) into the inferior olive of postnatal day 11 rats, waited $4 \mathrm{~d}$ for the dye to diff use, and cut sagittal sections. We imaged slices on an Olympus Optical (Tokyo, Japan) Fluoview confocal microscope.

\section{RESULTS}

Figure 1 illustrates anatomical differences between CFs and PFs at the light microscope level. By $15 \mathrm{~d}$ of age, each Purkinje cell receives a single climbing fiber input (Crepel et al., 1976; Ito, 1984), which wraps around the major dendritic branches of the
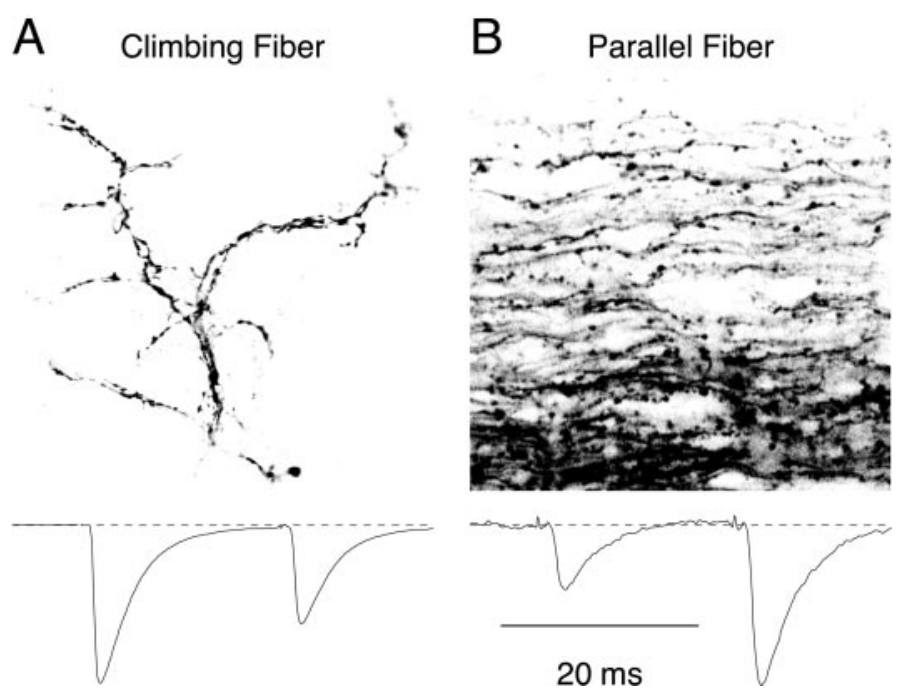

Figure 1. Overview of anatomical and physiological differences between cerebellar CFs and PFs. A, Top, Image of an individual CF labeled by injections of DiI in the inferior olive. The fiber is shown in a sagittal slice taken from cerebellar vermis, and a single optical section was imaged using a confocal microscope. The CF enters from the bottom and makes a complex arborization, with multiple processes running parallel to each other along the Purkinje cell dendrite, but the synapses themselves are not discernable. A, Bottom, Paired-pulse depression at the CF. A CF was stimulated twice at an interval of $20 \mathrm{msec}$, and the EPSCs were recorded in the Purkinje cell. The second EPSC is $60 \%$ of the size of the first. $B$, Top, Image of a band of PFs labeled with Texas Red dextran in a transverse slice (imaged using a confocal microscope). Each PF runs perpendicular to the Purkinje cell dendritic arbor. Varicosities, which correspond to synaptic contacts, are scattered along the length of the PF. $B$, Bottom, Paired-pulse facilitation at the PF. A band of PFs was stimulated twice at an interval of $20 \mathrm{msec}$, and the EPSCs were recorded in the Purkinje cell. The second EPSC is 2.5 times larger than the first. Physiology traces are adapted from Dittman et al. (2000).

Purkinje cell, making hundreds of contacts on somatic and dendritic spines (Fig. 1A). In contrast, PFs run perpendicular to the Purkinje cell dendritic arbor, with synaptic varicosities at $\sim 5 \mu \mathrm{m}$ intervals along their length, and they make few contacts with each Purkinje cell (Fig. 1B) (Palay and Chan-Palay, 1974).

Short-term plasticity in CFs and PFs are compared in Figure 1 (bottom). The CF shows paired-pulse depression, indicating a high probability of release, whereas the PF shows paired-pulse facilitation, indicating a low probability of release. To look for an anatomical basis for the difference in short-term plasticity and probability of release between CF and PF synapses, we examined CF and PF release sites using serial electron microscopy and quantified a number of synaptic characteristics that could play a role.

\section{Climbing fibers}

We prepared two series (of 108 and 143 sections) for examining release sites made by two CFs. We followed one CF in each series and identified the synaptic contacts made by each axon using the presence of presynaptic active zones and PSDs. Figure 2 shows four serial sections taken from two example CF synapses, in which we have identified and traced the CF (blue), the postsynaptic spines (pink), and ensheathing astrocytes (yellow). Both segments of axon have mitochondria present and a high density of vesicles. The release site in Figure $2 A$ is larger than average, whereas the release site in Figure $2 B$ is smaller than average. The spine in Figure $2 B$ (pink) connects through a very narrow neck to 

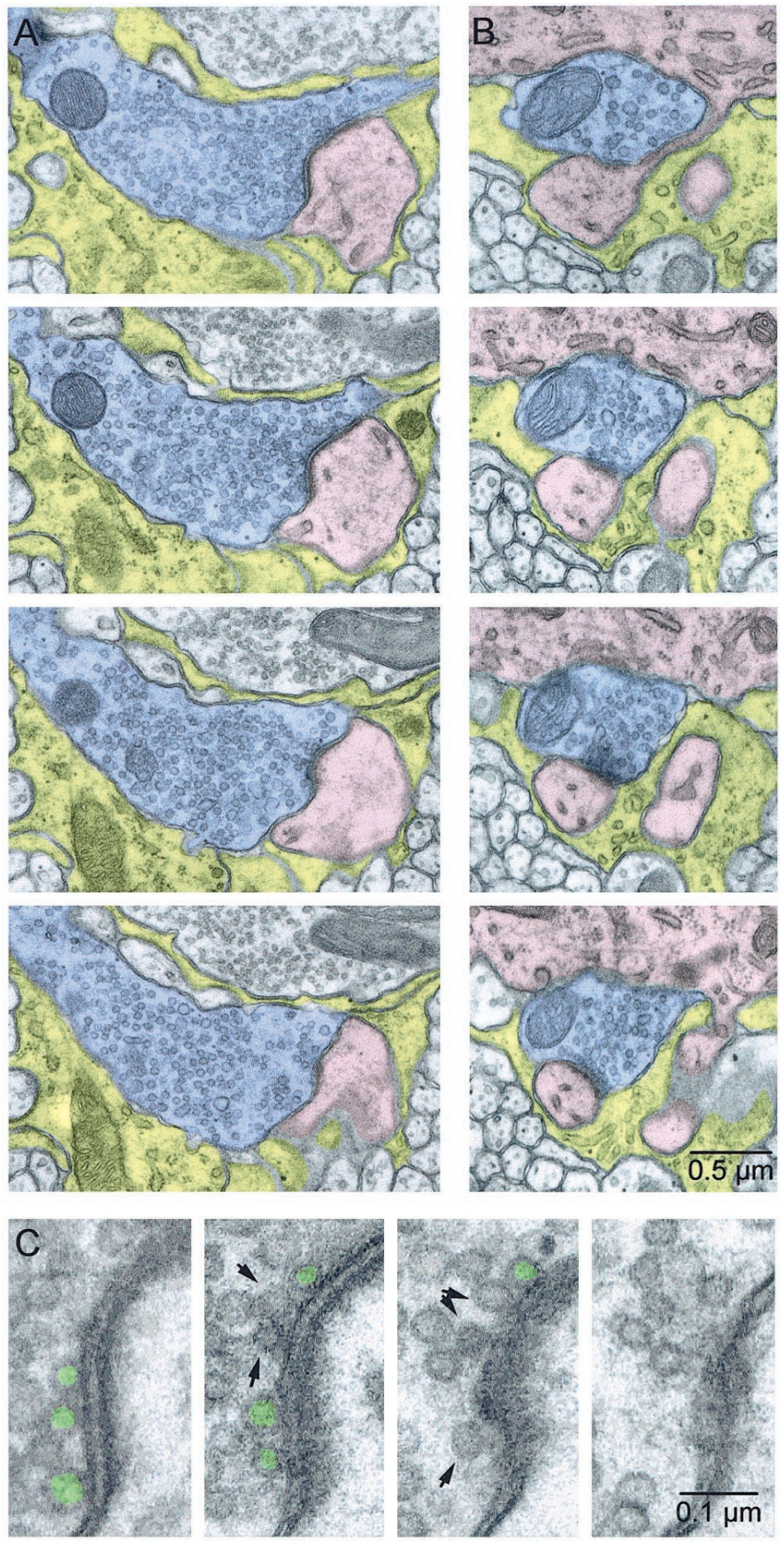

Figure 2. Serial EM sections of CFs. A, B, Two sample release sites from the same CF. CF axons are shaded blue, Purkinje cell dendrites and spines are shaded pink, and astrocytes are shaded yellow. The release site in $A$ is larger than average, whereas the release site in $B$ is smaller than average. In $A$, the synaptic cleft and PSD are clearly identifiable in the top three panels. In $B$, two spines are visible emerging from the parent dendrite at the top of each panel. The PSD for one spine is visible in the bottom three panels, and the second makes a PSD in later sections. $C$, Close-ups of the active zone in $A$. The presynaptic terminal is on the left, and the postsynaptic spine, with PSD clearly visible, is on the right. A vesicle was classified as docked (green) if it was located opposite a PSD and directly touched the presynaptic membrane. Nondocked vesicles close to the membrane are indicated by arrows. the parent dendrite (top). These two synapses were ensheathed by astrocytic processes (yellow) at both ends of the synaptic cleft. We classified vesicles as docked if they were located opposite PSDs in a spine and if the vesicle membrane directly touched the presynaptic membrane (Fig. $2 C$, green); if there was any cytoplasmic space between the two membranes, they were classified as nondocked (Fig. 2C, arrows). This strict criterion was used because only these docked vesicles are in a position to be readily released.

We made a partial reconstruction of one $\mathrm{CF}$ (Fig. $3 B, C$ ) and its postsynaptic Purkinje cell (Fig. $3 A, C$ ). Over this $\sim 60 \mu \mathrm{m}$ stretch of dendrite, we identified 67 release sites (Fig. 3, red), all of which were onto spines, and each spine received only one synapse. The $\mathrm{CF}$ wraps around the Purkinje cell dendrite, branching frequently and producing processes running back and forth over its surface to make clusters of contacts (Fig. 3C), similar to the light microscope view in Figure $1 A$. The location of synapses is not uniform over the Purkinje cell dendrite, because spines appear to form in clusters, and synapses do not occur at regular intervals along the CF.

We identified CF release sites using the presence of an active zone across from a PSD on a Purkinje cell spine. We made detailed reconstructions of 13 release sites in the first $\mathrm{CF}$ and 18 in the second. The reconstructed axon segments in Figure 4 illustrate various characteristics of these release sites. Some release sites were made en passant (Fig. $4 A-C$ ), and others were in distinct boutons (Fig. $4 A, D$ ). Multiple release sites frequently were found very near each other (Fig. $4 C$ ). Vesicle density near release sites was high, and often the density was lower in parts of the axon with no release sites (Fig. $A, D$ ). Most release sites had at least one mitochondrion nearby, and some had several (Fig. $4 A-D)$. Docked vesicles were distributed across the whole active zone (Fig. 4).

At these reconstructed release sites, we quantified the number of docked vesicles, PSD surface area, and spine volume. Because the averages from these series were nearly the same, we combined them (Table 1; see Fig. 7). We found that the number of docked vesicles ranged from 1 to 14 with an average of $7.3 \pm 3.0$ (mean \pm $\mathrm{SD})$ and that this number of docked vesicles at each site correlated with the PSD area $(r=0.80)$ and spine volume $(r=0.73)$.

To quantify the presence of mitochondria on the presynaptic side, we examined the 67 release sites in the reconstructed CF of Figure 3. When we measured the distance between the active zone and the nearest mitochondrion, we found that $84 \%$ of the release sites had a mitochondrion within $1 \mu \mathrm{m}$ (median, 0.32 $\mu \mathrm{m}$ ), and some release sites had as many as six mitochondria within $1 \mu \mathrm{m}$. Astrocytes are prominent over the proximal segment of the Purkinje cell dendrite (Fig. 2). We restricted our attention to the presence of astrocytic ensheathment around the perimeter of the synaptic cleft, quantifying the percentage of the perimeter that was bounded by astrocytic processes (same method as Ventura and Harris, 1999). A total of $58 \%$ of synapses were at least $90 \%$ ensheathed, and the median degree of ensheathment was $94 \%$ (average $87 \pm 18 \%$ ).

\section{Parallel fibers}

We prepared two series (of 89 and 82 sections) for examining the synapses made by PFs. PF synapses were easily identified as varicosities (Fig. $5 A, B$, blue), with a large increase in vesicle density around an active zone that contacted a spine with a postsynaptic density ( pink). Astrocytic processes ( yellow) appear to occupy less of the neuropil near PF synapses than near CF 

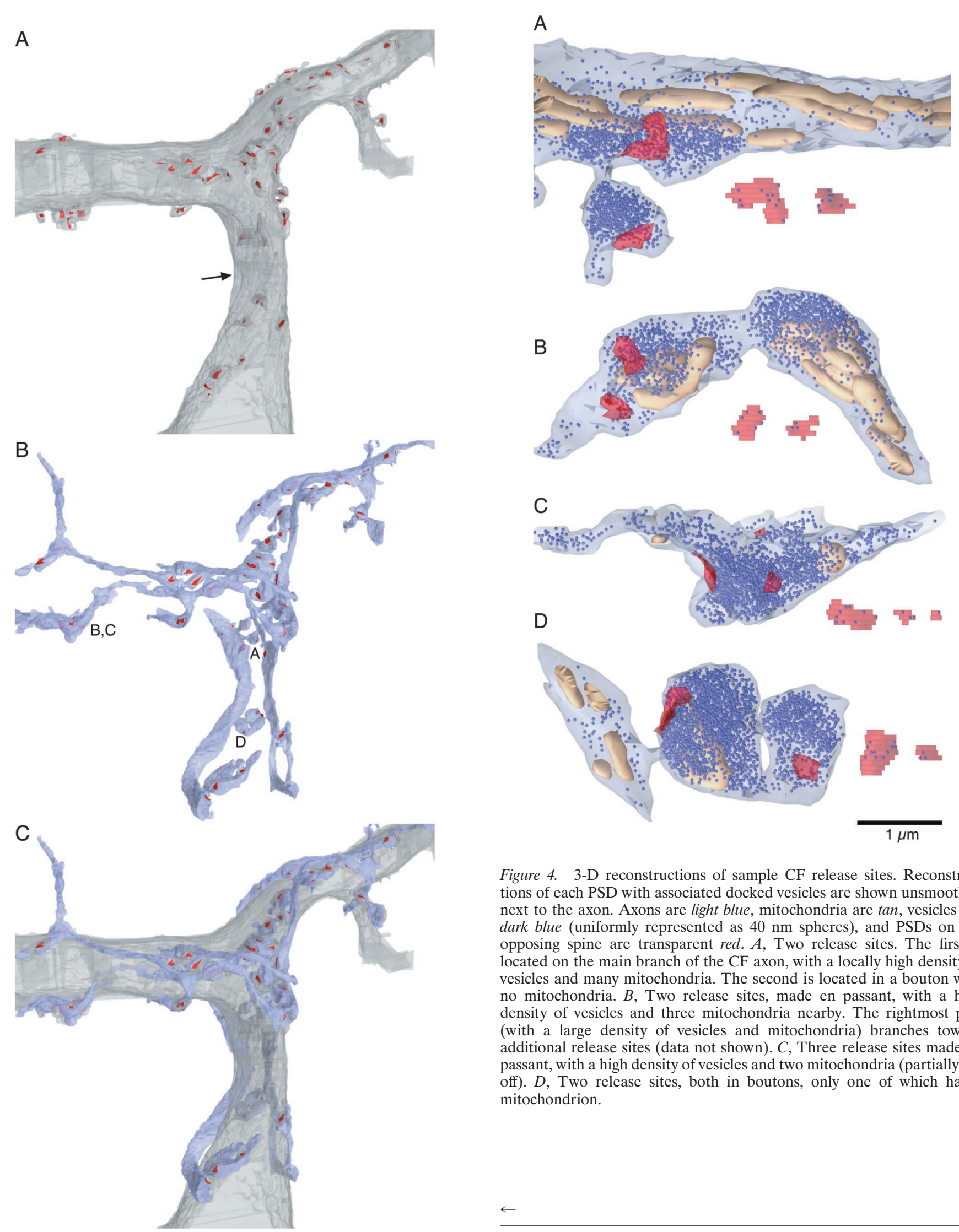

Figure 4. 3-D reconstructions of sample CF release sites. Reconstructions of each PSD with associated docked vesicles are shown unsmoothed next to the axon. Axons are light blue, mitochondria are tan, vesicles are dark blue (uniformly represented as $40 \mathrm{~nm}$ spheres), and PSDs on the opposing spine are transparent red. A, Two release sites. The first is located on the main branch of the CF axon, with a locally high density of vesicles and many mitochondria. The second is located in a bouton with no mitochondria. $B$, Two release sites, made en passant, with a high density of vesicles and three mitochondria nearby. The rightmost part (with a large density of vesicles and mitochondria) branches toward additional release sites (data not shown). $C$, Three release sites made en passant, with a high density of vesicles and two mitochondria (partially cut off). $D$, Two release sites, both in boutons, only one of which has a mitochondrion.

Figure 3. 3-D reconstruction of a CF terminating on a Purkinje cell from serial EM. A, Purkinje cell proximal dendrite. The soma is off the bottom, and distal into the molecular layer is toward the top. Spines that make contact with a CF are included, and their PSDs are labeled in red. The

dendrite is viewed at an angle, so as an indication of scale, the dendrite is $3.8 \mu \mathrm{m}$ in diameter at the position indicated by the arrow. $B$, CF axon, colored in blue. PSDs on the Purkinje cell are labeled in red. Labels $A-D$ in $B$ correspond to the segments of $\mathrm{CF}$ reconstructed in more detail in Figure 4. C, CF and Purkinje cell superimposed. 


\begin{tabular}{|c|c|c|}
\hline Characteristic & $\mathrm{CF}$ & $\mathrm{PF}$ \\
\hline Total vesicles per varicosity & - & $480 \pm 160(22)$ \\
\hline Docked vesicles per release site & $7.3 \pm 3.0(31)$ & $8.1 \pm 2.9(22)$ \\
\hline \multicolumn{3}{|l|}{ Mitochondria $<1 \mu \mathrm{m}$} \\
\hline (\% release sites) & $84 \%(67)$ & $67 \%(42)$ \\
\hline Ensheathment (median) & $94 \%(67)$ & $67 \%(42)$ \\
\hline PSD area $\left(\mu \mathrm{m}^{2}\right)$ & $0.14 \pm 0.08(31)$ & $0.13 \pm 0.04(22)$ \\
\hline Opposing spine volume $\left(\mu \mathrm{m}^{3}\right)$ & $0.20 \pm 0.09(35)$ & $0.13 \pm 0.03(22)$ \\
\hline
\end{tabular}

Values are mean $\pm \operatorname{SD}(n)$ or percentage of $(n)$.

synapses (compare with Fig. 2), although as quantified below, most PF synapses have at least some astrocytic ensheathment. The synapses in Figure 5, $A$ and $B$, had mitochondria in other sections that are not pictured. We classified vesicles as docked using the same strict criteria as for CFs (Fig. 5C, green).

We chose a set of 10 PFs in each series to reconstruct in detail. We identified release sites using the presence of an active zone across from a PSD on a Purkinje cell spine. Each spine received a single synaptic contact. All release sites were located in axonal varicosities, with high local vesicle densities (Fig. 6). Some release sites had nearby mitochondria (Fig. $6 A-C$ ), but others did not (Fig. 6D,E). Occasionally, we saw more than one release site per varicosity (Fig. $6 F$ ). Docked vesicles were distributed across the entire active zone (Fig. 6).

For these reconstructed synapses, we quantified the total number of vesicles in a varicosity, the number of docked vesicles, the PSD surface area, and the spine volume. The two series were not significantly different, so their results were combined (Table 1). The total number of vesicles in each varicosity was large (480 \pm 160, range of 237-770), similar to a previous study (Harris and Stevens, 1988). We did not perform a count of total vesicles at CF release sites, because their geometry is much more complex and they are not as readily isolated from each other. The PSD surface area was on average $0.13 \mu \mathrm{m}^{2}$ at the PF, which was similar to the average of $0.14 \mu \mathrm{m}^{2}$ at the $\mathrm{CF}$, but the variability was lower than at the $\mathrm{CF}$ (SD, 0.04 vs $0.08 \mu \mathrm{m}^{2}$ ) (Fig. $7 A$ ). In addition, the average number of docked vesicles was $8.1 \pm 2.9$ at PFs, which was close to the number at $\mathrm{CF}$ release sites $(7.3 \pm 3.0)$, and the distribution of the number of docked vesicles was also very similar for these two synapse types (Fig. 7B). The number of docked vesicles correlated with PSD area $(r=0.75)$.

All PFs that had release sites within the middle 20 sections $(1.2$ $\mu \mathrm{m})$ of the second series were reconstructed across the entire series to quantify the presence of presynaptic mitochondria and the degree of astrocytic ensheathment. Of the 39 parallel fibers in this population, four $(10 \%)$ had two release sites per varicosity, which were made onto different spines. This is considerably less than the $27 \%$ of varicosities with more than one release site reported in older rats (Harris and Stevens, 1988). Only one PF showed a second varicosity with a release site (2.2 $\mu \mathrm{m}$ away), consistent with the observation from fluorescent and Golgi staining that varicosities are spaced $5 \mu \mathrm{m}$ apart on average. Of the 39 PFs, $25(64 \%)$ had a mitochondrion within $1 \mu \mathrm{m}$ of the release site (Fig. 7C) and 11 (28\%) had no mitochondrion within the series. The median distance to the nearest mitochondrion was $0.37 \mu \mathrm{m}$, which was not greatly different from the CF $(0.32 \mu \mathrm{m})$. For 12 of 42 synapses (29\%), 90\% of the perimeter of the synaptic


Figure 5. Serial EM sections of PFs. A, B, Two example series. PFs are shaded blue, Purkinje cell spines are shaded pink, and astrocytes are shaded yellow. Every panel contains a clearly defined synaptic cleft and PSD. The release site in $A$ is larger than average, whereas the release site in $B$ is smaller than average. Although neither PF has a mitochondrion in the sections shown here, the release site in $A$ has a mitochondrion in a section that is $1.5 \mu \mathrm{m}$ away, and the site in $B$ has one in a section that is $0.2 \mu \mathrm{m}$ away. $C$, Close-up of the active zone in $A$. The presynaptic terminal is on the left, and the postsynaptic spine, with PSD clearly visible, is on the right. Docked vesicles are labeled in green, and nondocked vesicles close to the membrane are indicated by arrows. 

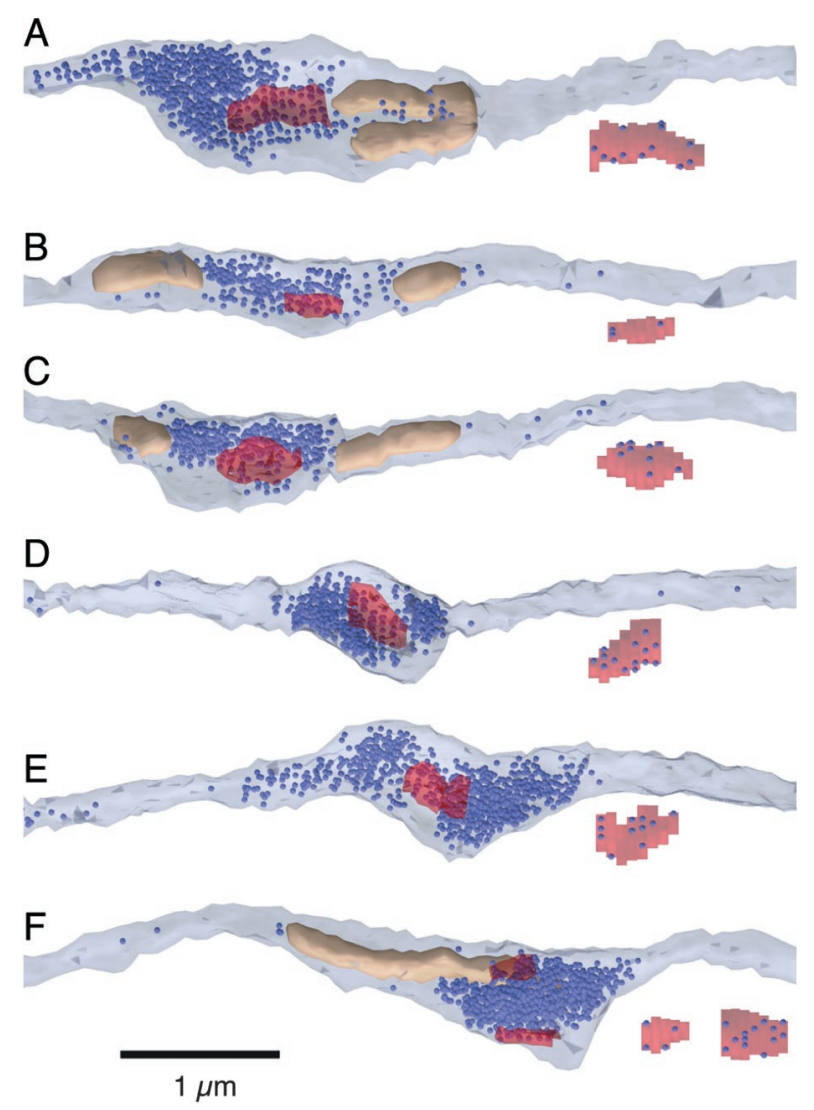

Figure 6. 3-D reconstructions of sample PF release sites. Reconstructions of each PSD with associated docked vesicles are shown unsmoothed under the axon. The color scheme is as described in the legend to Figure 4. $A-C, \mathrm{PFs}$ with one to two mitochondria near the release site. $D, E, \mathrm{PFs}$ without mitochondria. $F, \mathrm{PF}$ with two release sites onto different spines in the same varicosity.

cleft was ensheathed by astrocytes (median, 67\% ensheathed; average, $65 \pm 29 \%$ ) (Fig. $7 D$ ), which was less than at the CF.

\section{DISCUSSION}

These serial reconstructions of CF and PF synapses onto Purkinje cells revealed that, despite their distinctive overall anatomical structures, they were primarily similar in a number of ultrastructural characteristics related to synaptic physiology. Foremost among these similarities, these synapses have the same number of morphologically docked vesicles. Thus, the number of docked vesicles cannot account for the differences in the initial probability of release and short-term plasticity exhibited by CF and PF synapses.

\section{Probability of release}

We compared the numbers of docked vesicles and the electrophysiological properties of different synapses. Our measurements of seven to eight docked vesicles per active zone at the PF and CF are less than those reported for a number of cells in sensory pathways: 22 in goldfish bipolar neurons (von Gersdorff et al., 1996), 32 in frog saccular hair cells (Lenzi et al., 1999), and 130 in cat rod photoreceptors (Rao-Mirotznik et al., 1995). The large number of docked vesicles at these active zones may help these cells to respond rapidly and reliably to the sensory signal driving them. Each of these cells releases transmitter in response to graded or receptor potentials, so their probability of release cannot be compared.
A

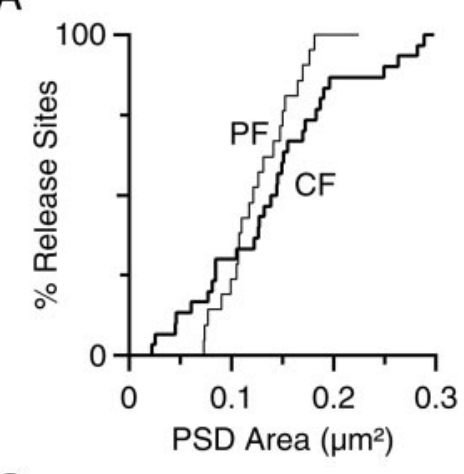

C

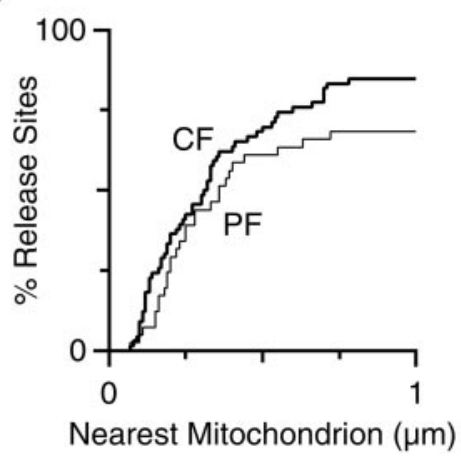

B

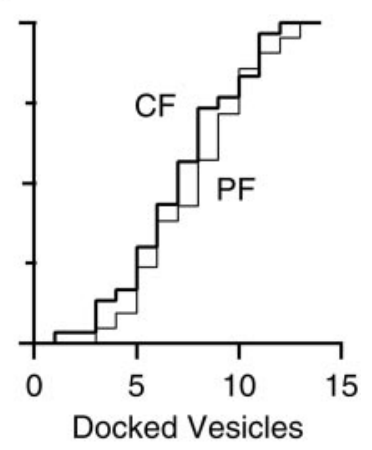

D

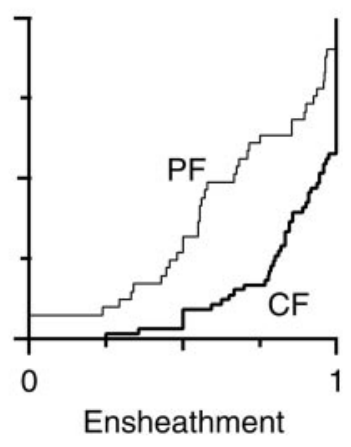

Figure 7. Comparison of ultrastructural characteristics at PF and CF release sites. Data are presented as cumulative histograms. PF distributions are shown as thin lines, and $\mathrm{CF}$ distributions are shown as thick lines. $A$, PSD area. $B$, Docked vesicle number. $C$, Distance from active zone to nearest mitochondrion. $D$, Degree of ensheathment.

A more direct comparison can be made with CNS neurons that fire action potentials and have a well defined probability of release. At hippocampal CA1 neurons, the number of docked vesicles per active zone can range from 2 to 36 (Harris and Sultan, 1995), with an average of 10 (Schikorski and Stevens, 1997). In layers $1 \mathrm{a}$ and $1 \mathrm{~b}$ of pyriform cortex, the average number of docked vesicles is 16 and 27, respectively (Schikorski and Stevens, 1999). These measurements, together with physiological studies of hippocampal and pyriform cortical cells, have suggested that the number of docked vesicles correlates with the probability of release (Bower and Haberly, 1986; Harris and Sultan, 1995; Dobrunz and Stevens, 1997; Murthy et al., 1997; Schikorski and Stevens, 1997, 2001). However, although there is a large difference in the probability of release between CF and PF synapses, they both have approximately the same number of docked vesicles, and this number is somewhat less that what has been observed at these other CNS synapses.

There are several possible explanations for why the number of docked vesicles at PF and CF synapses does not correlate with the probability of release. One possibility is that not all of the anatomically docked vesicles are release ready, which could arise from a priming step after docking that differs between PFs and CFs. Alternatively, there are many proteins involved in synaptic release, many of which have multiple isoforms or phosphorylation sites, which could affect probability of release (Sudhof, 1995). In addition, the calcium signal that drives release could differ between the two synapses as a result of differences in calcium influx, buffering, or diffusion distance to the release-ready vesicle. Such differences in release machinery or calcium signal would not be apparent ultrastructurally. 


\section{Depletion model of depression}

Our finding of multiple docked vesicles at CF release sites constrains models of the mechanisms underlying paired-pulse depression. One prominent model of PPD proposes that it is attributable to depletion of release-ready sites (Takeuchi, 1958; Elmqvist and Quastel, 1965; Betz, 1970). In the simplest case, a single vesicle is released from the RRP per release site, and the RRP corresponds to anatomically docked vesicles (Stevens and Wang, 1995). Our measurements of docked vesicles at the CF are inconsistent with this simple model, because the loss of a single vesicle from a pool of approximately eight vesicles cannot account for the observed PPD of $40 \%$ at the CF (Fig. $1 A$ ). One possibility is that not all anatomically docked vesicles are release ready, and our measurements of anatomically docked vesicles overestimate the RRP. Another possibility is that the RRP could be depleted more rapidly after the first pulse because of multivesicular release, as recent experiments at the CF suggest (Wadiche and Jahr, 2000).

\section{Other ultrastructural characteristics}

Although the primary focus of these studies was on docked vesicles, short-term plasticity, and probability of release, we also documented characteristics of these synapses that could affect other aspects of synaptic function: PSD area, mitochondrial distribution, and astrocytic ensheathment. PSD area was similar on average for both CF and PF synapses (0.14 vs 0.13$)$, but variability was larger at $\mathrm{CF}$ than PF synapses. This variability likely contributes to the distribution of miniature EPSC amplitudes at these synapses. For both types of synapses, a minority of active zones were $>1 \mu \mathrm{m}$ from the nearest mitochondrion (33\% of PFs, $16 \%$ of CFs). This may indicate heterogeneity in calcium handling and energy production, as has been suggested for CA3 synapses onto CA1 pyramidal cells, for which half of the varicosities lack mitochondria (Shepherd and Harris, 1998). We also found that both $\mathrm{PF}$ and $\mathrm{CF}$ synapses were ensheathed by astrocytic processes (average degree of ensheathment of $87 \%$ for CFs and $65 \%$ for PFs) to a greater extent than synapses in the stratum radiatum in area CA1 of the hippocampus (average of 43\%) (Ventura and Harris, 1999). Variability in cerebellar ensheathment has also been reported by Nusser et al. (2000). Because astrocytes play a large role in neurotransmitter uptake, these findings suggest that CF synapses are likely to be particularly insensitive to cross talk and desensitization because of spillover of glutamate.

\section{REFERENCES}

Betz WJ (1970) Depression of transmitter release at the neuromuscular junction of the frog. J Physiol (Lond) 206:629-644.

Bower JM, Haberly LB (1986) Facilitating and nonfacilitating synapses on pyramidal cells: a correlation between physiology and morphology. Proc Natl Acad Sci USA 83:1115-1119.

Brewer PA, Lynch K (1986) Stimulation-associated changes in frog neuromuscular junctions. A quantitative ultrastructural comparison of rapid-frozen and chemically fixed nerve terminals. Neuroscience 17:881-895.

Crepel F, Mariani J, Delhaye-Bouchaud N (1976) Evidence for a multiple innervation of Purkinje cells by climbing fibers in the immature rat cerebellum. J Neurobiol 7:567-578.

Dittman JS, Regehr WG (1998) Calcium dependence and recovery kinetics of presynaptic depression at the climbing fiber to Purkinje cell synapse. J Neurosci 18:6147-6162.

Dittman JS, Kreitzer AC, Regehr WG (2000) Interplay between facilitation, depression, and residual calcium at three presynaptic terminals. J Neurosci 20:1374-1385.

Dobrunz LE, Stevens CF (1997) Heterogeneity of release probability, facilitation, and depletion at central synapses. Neuron 18:995-1008.

Eccles JC, Llinas R, Sasaki K, Voorhoeve PE (1966) Interaction experiments on the responses evoked in Purkinje cells by climbing fibres. J Physiol (Lond) 182:297-315.

Elmqvist D, Quastel DMJ (1965) A quantitative study of end-plate potentials in isolated human muscle. J Physiol (Lond) 178:505-529.
Fiala JC, Harris KM (2001a) Extending unbiased stereology of brain ultrastructure to three-dimensional volumes. J Am Med Inform Assoc 8:1-16.

Fiala JC, Harris KM (2001b) Cylindrical diameters method for calibrating section thickness in serial electron microscopy. J Microsc 202:468-472.

Harris KM, Stevens JK (1988) Dendritic spines of rat cerebellar Purkinje cells: serial electron microscopy with reference to their biophysical characteristics. J Neurosci 8:4455-4469.

Harris KM, Sultan P (1995) Variation in the number, location, and size of synaptic vesicles provides an anatomical basis for the nonuniform probability of release at hippocampal CA1 synapses. Neuropharmacology 34:1387-1395.

Hashimoto K, Kano M (1998) Presynaptic origin of paired-pulse depression at climbing fibre-Purkinje cell synapses in the rat cerebellum. J Physiol (Lond) 506:391-405.

Herrera AA, Grinnell AD, Wolowske B (1985) Ultrastructural correlates of naturally occurring differences in transmitter release efficacy in frog motor nerve terminals. J Neurocytol 14:193-202.

Ito M (1984) The cerebellum and neural control. New York: Raven.

Konnerth A, Llano I, Armstrong CM (1990) Synaptic currents in cerebellar Purkinje cells. Proc Natl Acad Sci USA 87:2662-2665.

Lenzi D, Runyeon JW, Crum J, Ellisman MH, Roberts WM (1999) Synaptic vesicle populations in saccular hair cells reconstructed by electron tomography. J Neurosci 19:119-132.

Msghina M, Govind CK, Atwood HL (1998) Synaptic structure and transmitter release in crustacean phasic and tonic motor neurons. J Neurosci 18:1374-1382.

Murthy VN, Sejnowski TJ, Stevens CF (1997) Heterogeneous release properties of visualized individual hippocampal synapses. Neuron 18:599-612.

Nakajima Y, Reese TS (1983) Inhibitory and excitatory synapses in crayfish stretch receptor organs studied with direct rapid-freezing and freeze-substitution. J Comp Neurol 213:66-73.

Nusser Z, Roth A, Schorge S, Hausser M (2000) Simulations of synaptic transmission in 3-D reconstructions of cerebellar neuropil. Soc Neurosci Abstr 26:1122.

Palay SL, Chan-Palay V (1974) Cerebellar cortex: cytology and organization. New York: Springer.

Perkel DJ, Hestrin S, Sah P, Nicoll RA (1990) Excitatory synaptic currents in Purkinje cells. Proc R Soc Lond B Biol Sci 241:116-121.

Ramón y Cajal S (1995) Histology of the nervous system. New York: Oxford UP

Rao-Mirotznik R, Harkins AB, Buchsbaum G, Sterling P (1995) Mammalian rod terminal: architecture of a binary synapse. Neuron 14:561-569.

Rosenmund C, Stevens CF (1997) The rate of aldehyde fixation of the exocytotic machinery in cultured hippocampal synapses. J Neurosci Methods 76:1-5.

Schikorski T, Stevens CF (1997) Quantitative ultrastructural analysis of hippocampal excitatory synapses. J Neurosci 17:5858-5867.

Schikorski T, Stevens CF (1999) Quantitative fine-structural analysis of olfactory cortical synapses. Proc Natl Acad Sci USA 96:4107-4112.

Schikorski T, Stevens CF (2001) Morphological correlates of functionally defined synaptic vesicle populations. Nat Neurosci 4:391-395.

Shepherd GM, Harris KM (1998) Three-dimensional structure and composition of CA $3 \rightarrow$ CA 1 axons in rat hippocampal slices: implications for presynaptic connectivity and compartmentalization. J Neurosci 18:8300-8310.

Silver RA, Momiyama A, Cull-Candy SG (1998) Locus of frequencydependent depression identified with multiple-probability fluctuation analysis at rat climbing fibre-Purkinje cell synapses. J Physiol (Lond) 510:881-902.

Smith JE, Reese TS (1980) Use of aldehyde fixatives to determine the rate of synaptic transmitter release. J Exp Biol 89:19-29.

Stevens CF, Tsujimoto T (1995) Estimates for the pool size of releasable quanta at a single central synapse and for the time required to refill the pool. Proc Natl Acad Sci USA 92:846-849.

Stevens CF, Wang Y (1995) Facilitation and depression at single central synapses. Neuron 14:795-802.

Sudhof TC (1995) The synaptic vesicle cycle: a cascade of proteinprotein interactions. Nature 375:645-653.

Takeuchi A (1958) The long-lasting depression in neuromuscular transmission of frog. Jpn J Physiol 12:102-113.

Ventura R, Harris KM (1999) Three-dimensional relationships between hippocampal synapses and astrocytes. J Neurosci 19:6897-6906.

von Gersdorff H, Vardi E, Matthews G, Sterling P (1996) Evidence that vesicles on the synaptic ribbon of retinal bipolar neurons can be rapidly released. Neuron 16:1221-1227.

Wadiche JJ, Jahr CE (2000) Elevated levels of glutamate at cerebellar synapses. Soc Neurosci Abstr 26:1385.

Walrond JP, Govind CK, Huestis SE (1993) Two structural adaptations for regulating transmitter release at lobster neuromuscular synapses. J Neurosci 13:4831-4845. 